\title{
Questes
}

\section{Les Manganes de Constantin IX, un « paradis » au sein de Constantinople}

\section{Numa Buchs}

\section{(2) OpenEdition}

1 Journals

\section{Édition électronique}

URL : https://journals.openedition.org/questes/5822

DOI : $10.4000 /$ questes.5822

ISSN : 2109-9472

Éditeur

Les Amis de Questes

\section{Édition imprimée}

Date de publication : 30 juillet 2021

Pagination : 89-111

ISSN : 2102-7188

\section{Référence électronique}

Numa Buchs, «Les Manganes de Constantin IX, un « paradis » au sein de Constantinople », Questes

[En ligne], 43 | 2021, mis en ligne le 30 octobre 2021, consulté le 10 novembre 2021. URL : http:// journals.openedition.org/questes/5822 ; DOI : https://doi.org/10.4000/questes.5822

\section{(C) Association des amis de «Questes »}




\title{
Les Manganes de Constantin IX, un « paradis » au sein de Constantinople
}

\author{
Numa BUCHS \\ Sorbonne Université - Faculté des Lettres
}

S'intéresser aux jardins conduit à étudier un sujet d'une nature unique qui se distingue du caractère figé de l'architecture, des objets d'art ou même des textes. Les jardins, ensemble de plantes vivantes, se caractérisent en effet par un processus de changement permanent, variant au gré des saisons, mais aussi en fonction de la croissance et du déclin des végétaux. Les jardins, qui ont toujours été partie intégrante de la vie des Byzantins, offrent deux types d'avantages : le plaisir et l'utilité. En effet, les Byzantins utilisaient les espaces verts pour leurs loisirs, la production alimentaire ou même en vue d'asseoir leur prestige. Les études byzantines se sont majoritairement concentrées sur les jardins et les parcs de la plus haute catégorie sociale, à savoir les empereurs et l'aristocratie ${ }^{1}$.

Les byzantinistes se trouvent toutefois confrontés à la pauvreté des sources. Nous manquons notamment d'éléments matériels pour évaluer les dimensions et l'apparence des jardins byzantins de Constantinople.

\footnotetext{
${ }^{1}$ Pour les ouvrages fondamentaux, voir Antony Robert Littlewood, « The Scholarship of Byzantine Gardens », dans Byzantine Garden Culture, dir. Antony Robert Littlewood, Henry Maguire et Joachim Wolschke-Bulmahn, Washington, Dumbarton Oaks Research Library and Collection, 2002, p. 13-21. Sur les études des jardins constantinopolitains voir Antony Robert Littlewood, " Gardens of the Palaces », dans Byzantine Court Culture from 829 to 1204, dir. Henry Maguire, Washington, Dumbarton Oaks Research Library and Collection, 1997, p. 13-38 ; Nancy Patterson Sevcenko, "Wild Animals in the Byzantine Park », dans Byzantine Garden Culture, op. cit., p. 69-86.
} 
Ceux-ci ont tous disparu, pour la plupart enfouis sous des constructions postérieures et nous sont donc inaccessibles. Il nous est ainsi difficile de nous représenter les réalités, les formes ou encore le contenu de ces anciens espaces verts. Le moyen le plus évident pour parvenir à connaître des éléments matériels repose habituellement sur les fouilles archéologiques. Or, il est souvent impossible d'y recourir en raison de l'inaccessibilité des sites, sauf lors de grands travaux contemporains qui en favorisent l'excavation.

$\mathrm{Au}$ demeurant, cette absence permet d'éviter un écueil méthodologique lorsque l'on étudie les jardins de Byzance, à savoir restreindre le jardin à sa seule dimension matérielle, certes importante mais non exclusive ${ }^{2}$.

Privés en règle générale de vestiges, les historiens des jardins se tournent vers d'autres sources comme les sources narratives, hagiographiques, juridiques, voire les romans, afin de mieux comprendre comment les contemporains percevaient les jardins et les parcs de Constantinople ${ }^{3}$. Cela nous permet de nous intéresser à leur rôle et leurs représentations dans la société byzantine, en particulier à l'usage que les empereurs en faisaient grâce aux textes comme les ekphraseis ${ }^{4}$ et, dans le cadre de notre étude, à l'objectif politique de Constantin Monomaque (1042-1055) dans sa construction du jardin des Manganes.

Après le déclin urbain et économique du $\mathrm{VII}^{\mathrm{e}}$ et du VIII ${ }^{\mathrm{e}}$ siècle, le renouveau de Constantinople au IX ${ }^{\mathrm{e}}$ siècle a introduit une nouvelle demande pour des jardins d'agréments, et non plus simplement des espaces à vocation économique ou alimentaire. Les principaux acteurs de

\footnotetext{
${ }^{2}$ Joachim Wolschke-Bulmahn, «The Study of Byzantine Gardens : Some Questions and Observations from a Garden Historian », dans Byzantine Garden Culture, op. cit., p. 6-7.

${ }^{3}$ Henry Maguire, « Gardens and Parks in Constantinople », Dumbarton Oaks Papers, vol. 54, 2000, p. 251-252.

${ }^{4}$ Ibid., p. 260-262.
} 
ce renouvellement furent les aristocrates byzantins, bien que leurs empereurs aient conservé un intérêt pour les parcs. Cette tradition aristocratique s'est mêlée au $\mathrm{XI}^{\mathrm{e}}$ siècle aux conceptions impériales des jardins. En effet, à partir de 1028, plusieurs empereurs issus de l'aristocratie byzantine et parfois de lignées anciennes et prestigieuses, montèrent sur le trône grâce à l'impératrice de la dynastie macédonienne, Zoé (1028-1050), qui les épousa ou les adopta ${ }^{5}$. Devenus empereurs, ils conservaient l'intérêt aristocratique des jardins d'agrément ${ }^{6}$ mais devaient désormais l'intégrer à une conception davantage tournée vers la souveraineté, puisqu'ils étaient basileis des Romains. Leur arrivée au pouvoir ressuscita l'intérêt politique pour les jardins. La transition entre monde tardo-antique et byzantin du $\mathrm{VII}^{\mathrm{e}}$ au $\mathrm{VIII}^{\mathrm{e}}$ siècle avait en effet conduit à une raréfaction des jardins impériaux. Il fallut attendre le règne de Théophile (829-842) puis celui de Basile I ${ }^{\text {er }}$ (867-886) pour voir réapparaître des empereurs aménager des jardins ${ }^{7}$. C'est toutefois à partir des années 1030 et 1040 que l'aménagement des parcs impériaux devint véritablement important, avec un apogée sous Constantin IX.

Cet article aura pour objectif de s'interroger sur la manière dont la création de jardins impériaux, au cœur de Constantinople, a pu devenir un élément à part entière dans l'idéologie impériale d'un souverain byzantin au milieu du $\mathrm{XI}^{\mathrm{e}}$ siècle. Notre réflexion s'articulera autour des questions

\footnotetext{
${ }^{5}$ Michael Angold, The Byzantine Empire, 1025-1204. A Political History [1984], Londres/New York, Longman, 1997, p. 56-58.

${ }^{6}$ Sur les liens entre les familles aristocratiques et la fonction impériale, voir Alexander Kazhdan, "The Aristocracy and the Imperial Ideal », dans The Byzantine Aristocracy, IX to XIII centuries, dir. Michael Angold, Oxford, B.A.R., 1984, p. 45-52.

${ }^{7}$ Chronographiae quae Theophanis Continuati nomine fertur Libri I-IV, éd. Michael Featherstone, Juan Signes Codoñer et Karl de Boor, Berlin/Boston, De Gruyter, 2015, p. 128 ; Chronographiae quae Theophanis Continuati nomine fertur Liber quo Vita Basilii Imperatoris amplectitur, éd. Ihor Ševcenko, Berlin, De Gruyter, 2011, p. 280281. Théophane Continué est le premier chroniqueur byzantin à évoquer les constructions de jardins par les empereurs pour la période médiobyzantine.
} 
suivantes : à quel modèle le parc des Manganes peut-il correspondre? Quel était l'intérêt pour Constantin IX de se présenter en créateur d'un nouvel Éden au sein de la ville de Constantinople et quelles représentations les sources ont-elles conservées de cette œuvre horticole impériale?

\section{Un complexe végétal au cœur de Constantinople}

\section{Un parc dans la capitale}

$\mathrm{Au} \mathrm{XI}{ }^{\mathrm{e}}$ siècle, le développement urbain mené par les souverains byzantins s'est concentré sur deux régions principales, le quartier des Blachernes et l'Acropole. C'est cette dernière qui a le plus retenu l'intérêt de Constantin IX, puisque c'est à cet endroit qu'il a décidé d'édifier l'immense complexe des Manganes, devant remplir plusieurs fonctions. Ce complexe, qui contenait un jardin, est bien documenté par les sources byzantines, non seulement littéraires mais aussi $\operatorname{archéologiques~}^{8}$ et montre une évolution notable de l'intérêt des jardins pour les souverains byzantins, avant que cet engouement pour les aménagements horticoles ne soit mis de côté par les successeurs de Monomaque.

Constantin IX était en effet passionné par la plantation de végétaux et par des travaux de terrassements ${ }^{9}$ destinés à façonner des lieux selon

\footnotetext{
${ }^{8}$ Robert Demangel et Ernest Mamboury, Le quartier des Manganes (op. cit.) constitue la seule étude monographique sur les vestiges archéologiques du quartier des Manganes et qui inclut le complexe palatial du même nom. Voir aussi Wolfgang Müller-Wiener, Wolf Schiele, Renate Schiele, Bildlexikon zur Topographie Istanbuls: Byzantion, Konstantinupolis, Istanbul bis zum Beginn des 17. Jahrhunderts, Tübingen, Deutsches archäologisches Institut. Abteilung (Istanbul), 1977, p. 136-138 et le plan du quartier des Manganes p. 497.

${ }^{9}$ Byzance a conservé du monde romain sa capacité à transporter l'eau, en témoigne l'aqueduc de Valens qui est resté en état de fonctionnement, en dépit d'interruptions plus ou moins longues, du $\mathrm{IV}^{\mathrm{e}}$ à la fin $\mathrm{du} \mathrm{XIX}^{\mathrm{e}}$ siècle. L'irrigation byzantine prenait principalement la forme de simples canaux à ciel ouvert qui détournaient le précieux liquide des cours d'eau. Les empereurs avaient quant à eux les moyens de faire construire des canalisations enterrées qui pouvaient alimenter des citernes, elles aussi
} 
ses envies. Michel Psellos est l'auteur qui insiste le plus sur cette spécificité de Constantin IX dans le portrait qu'il dresse de lui dans son œuvre historique. Il y consacre plusieurs chapitres, pour souligner qu'il s'agissait, selon lui, d'amusements, voire de distractions indignes du rang impérial, mais qu'en dépit de tout, l'empereur s'y adonnait avec ferveur ${ }^{10}$.

L'inclination de l'empereur pour les éléments végétaux ainsi que les travaux entrepris par ce dernier pour les mettre en valeur sont décrits par Psellos comme une entreprise déraisonnable quand il raconte l'aménagement des alentours du complexe des Manganes. Sa description nous est parvenue grâce au récit de Michel Psellos, qui présente la construction de ce complexe, en particulier l'église et les jardins comme ayant contribué à épuiser les finances impériales ${ }^{11}$. Cette critique peut s'expliquer par la volonté de Psellos de montrer que l'empereur gaspillait l'argent public dans des projets de construction qui ne servaient pas à défendre les frontières de l'Empire. Le polymathe byzantin explique ainsi les désastres subis par Byzance à la fin du $\mathrm{XI}^{\mathrm{e}}$ siècle. L'impossibilité de payer un nombre suffisant de soldats est interprétée comme de la dilapidation des fonds impériaux par certains empereurs. Monomaque est présenté comme le plus représentatif de ces souverains dépensiers. En

enterrées. Il semble que les deux techniques aient été utilisées par Monomaque pour les Manganes. Psellos insiste sur la présence de canaux ouverts qui apportaient de l'eau et de la fraîcheur aux jardins des Manganes, Chronographie ou histoire d'un siècle de Byzance (976-1077), éd. Émile Renauld, Paris, Les Belles Lettres, 1926, t. II, 6, CLXXXVI. Le monde islamique a fait montre d'un niveau technique et d'une diffusion de l'irrigation bien supérieurs à ce dont Byzance a été capable de faire. En dépit de la proximité des deux mondes, il semble que Byzance n'ait guère tiré parti des avancées agricoles de ses voisins musulmans, en tout cas jusqu'au XI ${ }^{\mathrm{e}}$ siècle. Voir Michel Kaplan, Les hommes et la terre à Byzance du VI au XI siècle : propriété et exploitation du sol, Paris, Publications de la Sorbonne, 1992, p. 67-69.

${ }^{10}$ Psellos, Chronographie, éd. cit.

${ }^{11}$ Sur la question des éléments politiques dans l'œuvre de Psellos, voir Milena Repajic, "The Political Thought of Psellos in the Chronographia: The Wise Advisor, the Clever General, and the City », Limes Plus, vol. 13, n², 2016, p. 55-86. 
dépit du parti pris de l'auteur, son récit n'en reste pas moins inestimable, puisqu'il était un contemporain des travaux et un conseiller de Constantin Monomaque, autrement dit, un témoin de choix.

Nous pouvons vérifier certaines des indications de Psellos grâce à l'existence de témoignages postérieurs ${ }^{12}$ et de fouilles archéologiques ${ }^{13}$, et nous pouvons remarquer que la description des jardins effectuée par Psellos était véridique. De plus, ces autres témoignages confirment que les Manganes occupaient une place particulière au sein des jardins impériaux.

L'empereur avait fait planter de nombreuses fleurs ${ }^{14}$ afin de constituer des étendues de prairies fleuries pour embellir les espaces entourant son église, à l'intérieur même des limites du complexe. Le plus impressionnant était toutefois l'agencement même des jardins, dont une partie fut conçue pour être suspendue ${ }^{15}$, c'est-à-dire que des arbres avaient été plantés sur des terrasses en hauteur, tandis que d'autres

${ }^{12}$ Raymond Janin, La géographie ecclésiastique de l'Empire byzantin. Première partie. Le siège de Constantinople et le patriarcat æecuménique, tome III, Les églises et les monastères, Paris, Centre National de la Recherche Scientifique, 1953, p. 7680.

${ }^{13}$ Robert Demangel et Ernest Mamboury, Le quartier des Manganes, op. cit., p. 2337.

${ }^{14}$ L'usage du végétal, comme élément du pouvoir, date de bien avant le $\mathrm{XI}^{\mathrm{e}}$ siècle, mais il semble que le règne de Monomaque ait été un apogée de son utilisation pour magnifier la fonction impériale. En témoignent non seulement la création du jardin des Manganes, mais aussi la présence d'éléments végétaux parmi les commandes impériales qui ont survécu jusqu'à nous. La couronne de Monomaque conservée à Budapest montre, en effet, la représentation de l'empereur enserrée par des vignes, tout comme les images des impératrices Zoé et Théodora, qui l'entourent, tandis que les personnifications féminines qui les encadrent sont, chacune, flanquées de deux cyprès. Voir Etele Kiss, "The State of Research on the Monomachos Crown and Some Further Thoughts ", dans Perceptions of Byzantium and its neighbours : 8431261, dir. Olenka Z. Pevny, New York/New Haven, The Metropolitan Museum of Art/Yale University Press, 2000, p. 70-71.

${ }^{15}$ Le parallèle avec les jardins suspendus de Babylone, célèbre merveille du monde, est évident. L'élément spectaculaire ne pouvait que plaire à Monomaque, mais cette décision offrit à Psellos un angle d'attaque lui permettant de présenter les terrasses de Monomaque, constructions coûteuses et superficielles, comme un symbole de la vanité de l'empereur. 
l'avaient été sur des gradins qui redescendaient en pente depuis les murailles maritimes vers la plaine, où se trouvaient les autres bâtiments ${ }^{16}$. Enfin, pour l'agrément mais aussi pour assurer l'approvisionnement en eau de cet écrin végétal, l'empereur ne négligea pas de faire creuser des canaux et d'aménager des fontaines ${ }^{17}$. En effet, des installations complexes d'irrigation furent mises en place afin d'approvisionner les jardins des Manganes en eau, comme le démontrent plusieurs vestiges de canalisations ainsi que des citernes ${ }^{18}$.

Constantin IX a dépensé sans compter pour ces jardins, et même Psellos a dû reconnaître qu'il s'agissait d'un ensemble particulièrement bien agencé et d'une grande beauté ${ }^{19}$. Il est évident que le jardin des Manganes présentait un contraste frappant avec le Mésoképion, le

\footnotetext{
${ }^{16}$ Psellos, Chronographie, éd. cit., t. II, 6, CLXXXVI.

${ }^{17}$ À partir du IX ${ }^{\mathrm{e}}$ siècle, les empereurs renouèrent avec la tradition antique et christianisée d'installation de jardins, de fontaines et de travaux d'adduction d'eau. Cette renaissance culturelle peut être discernée dans les arts et la littérature. Voir Paul Magdalino, "The Culture of Water in the "Macedonian Renaissance" ", dans Fountains and Water Culture in Byzantium, dir. Brooke Shilling et Paul Stephenson, Cambridge, Cambridge University Press, 2016, p. 130 et 144. Ce développement du thème de l'eau et de son champ lexical parmi les élites lettrées de Constantinople apparaît clairement sous Monomaque dans la poésie, où l'empereur est présenté comme un nouveau «Pactole» ou encore un autre «Nil», fleuve dispensateur de richesses et de dignités. Voir Ingela Nilsson, «Words, Water and Power : Literary Fountains and Metaphors of Patronage in Eleventh and Twelfth-Century Byzantium », dans Fountains and Water Culture, op. cit., p. 268-269.

${ }^{18}$ Henry Maguire, "Gardens and Parks in Constantinople », art. cit., p. 260. Les seules sources archéologiques qui nous restent sont deux citernes et les fondations des murs d'une partie des terrasses des jardins. Ces éléments nous sont uniquement accessibles grâce aux photos prises par la mission de recherche installée à Istanbul lors de l'occupation alliée de 1921 à 1923. Voir Robert Demangel et Ernest Mamboury, Le quartier des Manganes et la première région de Constantinople, Paris, De Boccard, 1939, deuxième fascicule, p. 2-5.

${ }^{19}$ Psellos, Chronographie, éd. cit., t. II, 6, CLXXXVI. Il faut cependant se méfier de son assertion sur la superficie des prairies que l'empereur avait fait aménager pour relier les différents bâtiments qui constituaient le complexe des Manganes, puisque l'auteur indique qu'elles ne pouvaient pas être contemplées dans leur globalité avec facilité. Il s'agit d'une exagération de Psellos pour souligner, là encore, le gâchis des ressources de l'État dans un projet considéré comme non essentiel pour la défense des frontières.
} 
principal jardin impérial, situé à l'intérieur du Grand Palais, seul autre grand jardin impérial bien connu par les sources ${ }^{20}$.

Le site était organisé sur deux niveaux. Le plus bas, qui correspondait au terrain qui avait conservé sa hauteur précédant les travaux de Monomaque, était large de près de deux cent cinquante mètres à l'extrémité sud, mais se rétrécissait progressivement en remontant vers le nord. Cette zone était bordée à l'est par les terrasses et les gradins boisés du parc et par une haute terrasse à l'ouest qui donnait sur la ville. Le monastère et le palais ont été édifiés à ce niveau, à proximité du mur maritime. Nous savons avec certitude que des portions de la terrasse occidentale furent construites pendant le règne de Constantin Monomaque, mais il semble bien que des éléments architecturaux plus anciens aient été réemployés dans le projet de l'empereur. Le mur qui fermait le complexe des Manganes du côté de la ville faisait près de six cents mètres de long ${ }^{21}$. Au niveau de la partie basse, la plus spacieuse, on trouvait une vaste zone, adaptée aux chevauchées. Elle était d'ailleurs encore utilisée pour la pratique des sports équestres durant la période ottomane.

La partie haute correspondait, quant à elle, à la zone surélevée par plusieurs terrasses et gradins à la suite des travaux de Constantin IX. Ce dernier y avait fait planter des bosquets d'arbres qui apparaissaient suspendus dans les airs, comme l'indique Psellos, et qui dépassaient des remparts maritimes. Cette zone haute était large de près de

\footnotetext{
${ }^{20}$ Henry Maguire, «Imperial Gardens and the Rethoric of Renewal », dans New Constantines. The Rhythm of Imperial Renewal in Byzantium, $4^{\text {th }}-13^{\text {th }}$ Centuries, Papers from the Twenty Sixth Spring Symposium of Byzantine Studies, St Andrews, March 1992, dir. Paul Magdalino, Aldershot, Variorum, p. 181-198, ici p. 182.

${ }^{21}$ Henry Maguire, « Gardens and Parks in Constantinople », art. cit., p. 260-262.
} 
cent quatre-vingt-dix mètres dans sa partie méridionale et beaucoup plus étroite au fur et à mesure qu'elle remontait vers le nord ${ }^{22}$.

Parmi les constructions des jardins, nous avons conservé deux citernes construites en contiguïté avec la terrasse au niveau sud du complexe. Bien que ces citernes datent d'une époque plus ancienne, probablement protobyzantine, celle située la plus au sud montre des traces de réparations médiévales, sans doute à l'époque de Constantin IX. Ces citernes, d'une certaine ampleur, qui ont survécu prouvent l'importance des réservoirs d'eau qui pouvaient récupérer l'eau de pluie et de l'eau apportée par les aqueducs, comme celui de Valens. Elles constituaient des réserves qui assuraient l'approvisionnement des bains et l'irrigation des végétaux qui faisaient le délice de l'empereur ${ }^{23}$.

La zone toute entière était extrêmement longue. Le site du complexe des Manganes, englobant les jardins, se courbait vers le nord-ouest le long d'une élévation de terrain, puisqu'il était adossé à l'Acropole. Les contraintes du terrain faisaient que le complexe était beaucoup plus large dans la partie sud que dans la partie nord. Le parc n'avait donc pas une forme symétrique ou même régulière, mais s'apparentait à une défense d'éléphant qui longeait le mur maritime vers la Corne d'Or.

Il était donc impossible de voir l'ensemble du jardin en se postant à l'une de ces extrémités. Psellos n'exagérait pas sur ce point, même s'il faut reconnaître qu'il s'agissait d'une illusion d'optique puisque, si le visiteur se plaçait contre le mur maritime, il aurait été capable de voir l'intégralité des jardins ${ }^{24}$. Il faut aussi prendre en compte le fait que les bâtiments du complexe des Manganes pouvaient empêcher de contempler

\footnotetext{
${ }^{22}$ Ibid., p. 261.

${ }^{23}$ Sur la question des fontaines dans les loisirs des Byzantins, voir Fountains and Water Culture in Byzantium, op. cit.

${ }^{24}$ Henry Maguire, « Gardens and Parks in Constantinople », art. cit., p. 261.
} 
le parc dans son intégralité, sauf en se plaçant sur la terrasse la plus haute. Cette illusion avait pour vocation à présenter au visiteur un jardin interminable, reflet de la toute-puissance de l'empereur et rappel du caractère infini de l'Éden auquel les Manganes devaient ressembler. La possibilité de contempler l'intégralité des jardins en accédant aux gradins les plus élevés et de se rendre compte de cette supercherie était réservée aux proches et aux invités de l'empereur, rappelant la hiérarchisation de la société et de la cour byzantines.

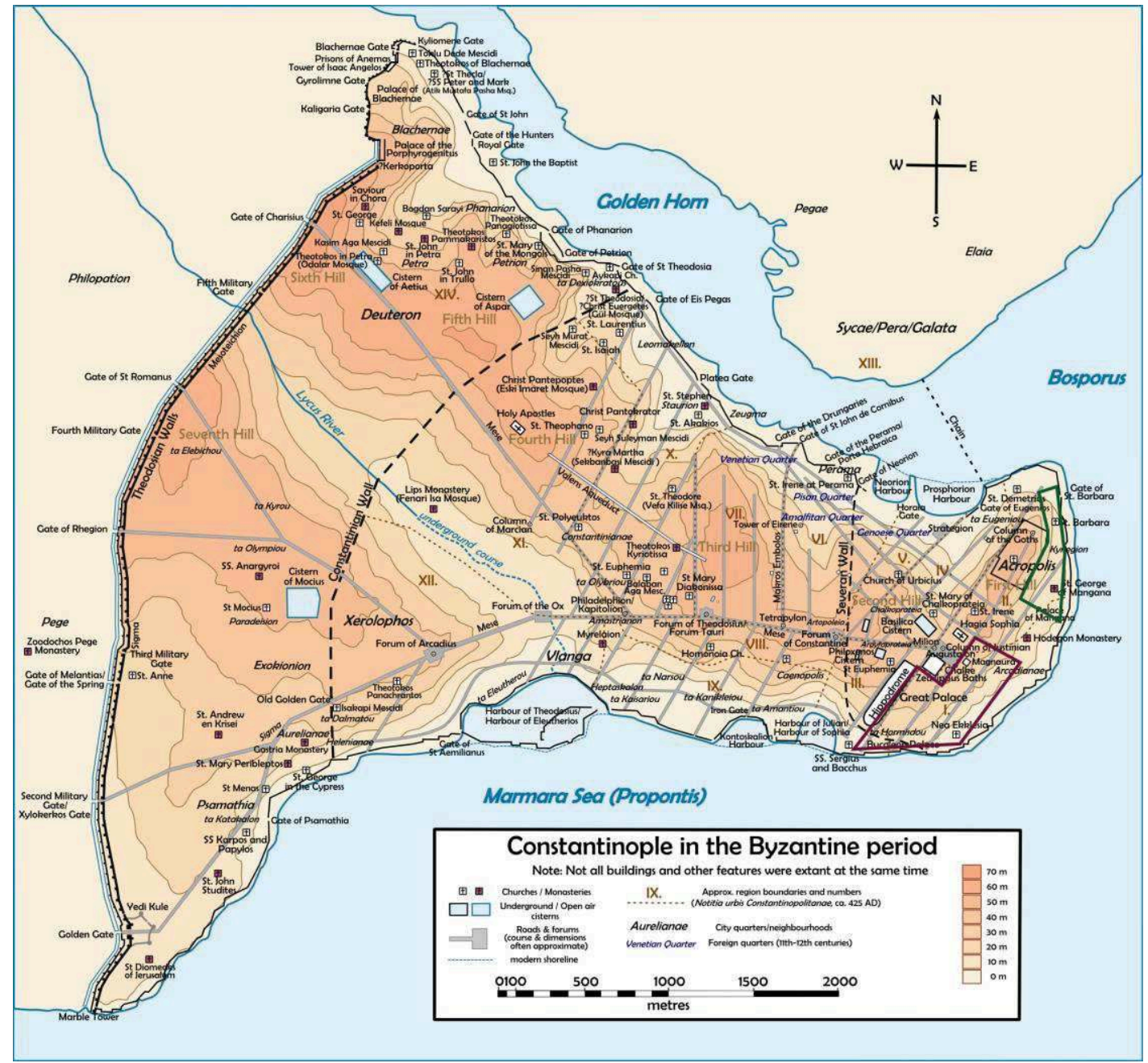

Fig. 1 : Plan de Constantinople présentant les principaux monuments politiques et religieux à l'époque du règne de Constantin Monomaque. On peut y voir la délimitation approximative des jardins des Manganes (au nord) et celle de l'ensemble du complexe palatial y compris le terrain de polo et le jardin du Mésoképion (au sud). On peut noter l'étendue géographique des jardins par rapport au Grand Palais ${ }^{25}$.

${ }^{25}$ Crédit : https://fr.wikipedia.org/wiki/Constantinople\#/media/File:Byzantine_Consta ntinople-en.png, image modifiée par l'auteur. 
La taille représentait un enjeu dans les constructions impériales, qu'il s'agisse de jardins ou de bâtiments. L'échelle de l'église et du parc des Manganes n'a pas échappée à cette règle, en particulier quand on compare l'étendue du jardin du Mésoképion avec celle des Manganes. En effet, le premier était petit et confiné dans les limites du complexe du Grand Palais, alors que le second était vaste et situé en-dehors de l'ensemble palatial qui abritait la majeure partie des constructions politiques des basileis au sein de Constantinople ${ }^{26}$. La localisation du projet de Constantin IX tend à montrer qu'il souhaitait se démarquer de ses prédécesseurs et inscrire sa marque dans la topographie de la ville de façon évidente. Le Mésoképion était situé au sein de la principale partie du palais, tandis que les jardins des Manganes se trouvaient sur une haute terrasse au nord-est du palais et dans un lieu extérieur, sur une élévation, qui donnait aux terrasses une impression plus céleste que terrestre. Cet éloignement du jardin des Manganes par rapport au palais permettait de souligner le caractère personnel de la création de l'empereur, et il fallait aussi prendre en compte la nécessité de disposer de terrains en quantité suffisante. On peut ainsi noter que le jardin des Manganes était enclos par un mur ${ }^{27}$, puisque Psellos précise que l'un des soins de l'empereur avait été de l'entourer d'une enceinte, dont nous avons retrouvé les fondations. La décision de ceindre les jardins des Manganes d'une barrière permettait de couper le complexe d'un contact direct avec le reste de la ville, ce qui convenait parfaitement à ses deux attributions, aussi bien politiques que monastiques. Enclore un espace vert était une spécificité élémentaire du jardin monastique, voire du jardin byzantin traditionne ${ }^{28}$.

\footnotetext{
${ }^{26}$ Ibid., p. 262.

${ }^{27}$ Henry Maguire, « Gardens and Parks in Constantinople », art. cit., p. 260.

${ }^{28}$ Veronica della Dora, Landscape, Nature, and the Sacred in Byzantium, Cambridge, Cambridge University Press, 2016, p. 108. C'est une habitude prise par les Byzantins
} 


\section{Un espace plurifonctionnel}

Le jardin des Manganes revêtait deux fonctions : celle de jardin palatial byzantin, mais aussi d'espace monastique. En effet, si le complexe avait pour vocation d'abriter l'empereur et sa nouvelle école de droit, Monomaque n'oublia pas de placer également au cœur de son projet un monastère et l'église qui le desservait. La volonté de Constantin IX de faire planter de nombreux arbres fruitiers, parmi lesquels des pommiers et des poiriers ${ }^{29}$, nous indique que le basileus voulait assurer une source d'alimentation aux résidents du Tropaiophore, qu'ils soient étudiants, patients ou moines. En choisissant de tels plants, le fondateur assurait à son oikos une source d'approvisionnement qui lui était intégrée et qui lui appartenait en propre, mais aussi de revenus, puisque les excédents des récoltes de fruits pourraient être vendus. Enfin, cela permettait d'assurer les principes d'autarcie et d'autosuffisance induits par la référence au jardin d'Éden. Un paradis terrestre, reflet du jardin de Dieu, se devait d'abriter de tels arbres ${ }^{30}$. Leur absence aurait sans doute affaibli la symbolique de la création impériale. D'autant plus que la construction du complexe était à l'origine un monastère dont la fondation reposait sur le principe d'autarcie, y compris alimentaire. On peut d'ailleurs noter que la tradition à Byzance voulait que l'on plante un jardin ou un verger avant d'édifier le monastère lui-même ${ }^{31}$.

Les jardins monastiques étaient d'une importance fondamentale dans la culture byzantine. Ils avaient une place essentielle dans l'existence des monastères et, partiellement exploités comme potagers, ils

à partir de la fin du $\mathrm{VII}^{\mathrm{e}}$ siècle, qui décidèrent de ne plus réaliser de jardins complètement ouverts, mais préféraient aménager une enceinte qui entourait le jardin, de la même manière que le jardin entourait la demeure ou le monastère.

${ }^{29}$ Psellos, Chronographie, éd. cit., t. II, 6, CCI.

${ }^{30}$ Veronica della Dora, Landscape, Nature, and the Sacred in Byzantium, op. cit., p. 108.

${ }^{31}$ Ibid., p. 110. 
fournissaient à la communauté une part consistante de son alimentation ${ }^{32}$. Ils assuraient la pérennité des monastères puisqu'on y retrouvait des vignobles, des légumes, des fruits et même des herbes. Le travail manuel étant perçu comme une forme de méditation, s'occuper des plantations du monastère remplissait d'ailleurs une bonne part des journées des moines et des moniales. De plus, l'hôpital des Manganes, installé au sein du complexe, avait sans aucun doute besoin d'un jardin capable de lui fournir les herbes lui permettant de produire des remèdes ${ }^{33}$. Enfin, les monastères étaient des éléments centraux de la société byzantine dont l'empereur devait se faire le protecteur en leur assurant des moyens de subsistance, et ce d'autant plus s'il s'agissait d'une fondation impériale.

Les Manganes étaient aussi un jardin palatial, desservant un palais que l'empereur se fit construire. Ce fut une véritable résidence impériale puisque Monomaque y a vécu, y a tenu sa cour et y mourut en janvier 1055. Or les jardins palatiaux avaient une vocation tout à fait différente des jardins monastiques. Extension du palais de l'empereur, ces jardins servaient principalement à impressionner les visiteurs et faisaient partie de l'apparat habituel qui devait entourer un basileus ${ }^{34}$. Ils étaient principalement composés de fontaines, d'arbres et de mosaïques. La conception du jardin des Manganes relevait donc d'un mélange de deux aspects différents.

\footnotetext{
32 Alice-Mary Talbot, «Byzantine Monastic Horticulture : The Textual Evidence », dans Byzantine Garden Culture, op. cit., p. 37-67. Pour une comparaison avec le monde monastique occidental, voir Joëlle Fuhrmann, «Les différentes sources, caractéristiques et fonctions des jardins monastiques au Moyen Âge », dans Vergers et jardins dans l'univers médiéval, Aix-en-Provence, PUP, 1990, p. 115-119; Bernard Beck, " Jardin monastique, jardin mystique. Ordonnance et signification des jardins monastiques médiévaux », Revue d'histoire de la pharmacie, t. 327, 2000, p. $380-388$.

${ }^{33}$ Alice-Mary Talbot, «Byzantine Monastic Horticulture : The Textual Evidence», art. cit., p. 54.

${ }^{34}$ Henry Maguire, «Imperial Gardens and the Rethoric of Renewal », art. cit., p. 181198.
} 
La gestion des Manganes n'est pas clairement établie et le partage des différentes parties du parc ne peut pas être démontré. Habituellement, c'étaient les moines qui assuraient l'entretien des jardins dépendant de leur monastère ou, le cas échéant, le confiaient à des salariés laïcs, mais la présence de l'empereur et son utilisation des jardins rendent incertaine cette solution. Il est plus probable que l'entretien des jardins ait été assuré grâce aux ressources concédées au complexe du Tropaiophore ${ }^{35}$, même si les moines pouvaient y jouer un rôle. La partition de ceux-ci entre les parties publiques, en particulier celles accessibles aux malades de l'hôpital, aux étudiants de l'école de droit et aux moines, et celles réservées à l'empereur ne peut pas être identifiée avec certitude, mais nous savons qu'elle existait puisque l'empereur et la cour n'auraient pas accepté que l'ensemble des parties soient accessibles au public. Cette partition était nécessaire pour assurer la séparation indispensable entre espaces impériaux et monastiques. Elle était, également, pour les moines, une condition préalable pour assurer leur tranquillité et leur éviter les distractions du monde extérieur. De même pour le cérémonial impérial, l'espace de vie et de représentation de l'empereur réclamait une démarcation avec les autres lieux publics pour souligner la sacralité et la souveraineté du basileus.

Psellos nous livre la clé de ce qu'il présente comme un entêtement impérial dans ces travaux. Il indique que la beauté de chaque élément construit, installé ou planté dans les Manganes avait pour fonction de sublimer l'harmonie des autres. Les prairies fleuries, ainsi que les jeux d'eau et de lumières attiraient l'œil au point que le visiteur était complètement absorbé par la contemplation des constructions et des

35 Nicolas Oikonomidès, «St Georges of Mangana, Maria Skleraina, and the "Malyjsion" of Novgorod», Dumbarton Oaks Papers, vol. 34-35, 1980-1981, p. 241-242. 
jardins des Manganes ${ }^{36}$. Lorsque Psellos écrit les chapitres concernant Constantin IX, au début des années 1060, les jardins devaient encore être bien entretenus et avoir conservé la forme qu'ils avaient pendant le règne du basileus ${ }^{37}$. Il lui aurait donc été difficile de nier l'esthétisme du jardinmais, bien au contraire, le caractère remarquable de celui-ci lui permettait de critiquer Monomaque pour la débauche de moyens utilisés pour sa construction.

\section{Le créateur de l'Éden : bon empereur ou mauvais empereur}

\section{Une passion critiquée par Psellos}

Psellos attribue à Constantin Monomaque une véritable obsession pour les arts du jardin. Il indique que l'empereur ne pouvait pas tolérer l'existence d'éléments naturels non-organisés par ses soins, en particulier si l'endroit avait été aride auparavant. Toutefois derrière ces descriptions exagérées, il faut sans doute voir la volonté de Monomaque de transformer une zone perçue comme désertique, ou peu favorable à la végétation, en un endroit luxuriant couvert d'arbres fruitiers, déracinés et replantés, pour constituer un verger rapidement afin d'accélérer la constitution de son jardin. Des mottes de terres fertiles étaient disposées pour favoriser l'apparition et le renouvellement de la végétation ${ }^{38}$. Évidemment ces travaux étaient accompagnés par des ouvrages d'irrigation pour assurer la pérennité de tant de labeur.

\footnotetext{
${ }^{36}$ Psellos, Chronographie, éd. cit., t. II, CLXXXVII. Il peut s'agir là encore d'une amplification de Psellos qui voulait souligner l'utilisation de grandes richesses pour le projet de Monomaque, alors qu'il aurait pu en faire un usage plus avisé.

${ }^{37}$ Les jardins des Manganes s'étendaient sur près de huit cents mètres dans le sens nord-sud, de la pointe de Seraglio au mur maritime de la ville et atteignait une largeur maximale de deux cent cinquante mètres, voir Henry Maguire, « Gardens and Parks in Constantinople », art. cit., p. 260.

${ }^{38}$ Psellos, Chronographie, éd. cit., t. II, 6, CLXXIV.
} 
Psellos voyait d'un mauvais œil les habitudes de l'empereur qui dépensait tant de temps et d'argent pour construire un parc de cette envergure. Il considérait la construction d'un complexe qui n'avait pas pour vocation à défendre l'Empire byzantin comme peu appropriée avec les fonctions d'un bon empereur ${ }^{39}$. Pour souligner le caractère de la démesure de Monomaque, il le présentait comme une sorte d'imitateur de Dieu en cherchant à réaliser un bouleversement de la nature. Cette comparaison avait pour vocation à souligner la présomption de l'empereur qui se livrait à une forme de compétition avec Dieu. Si toute construction, impériale ou non, conduisait forcément à transformer la nature, modifiant ainsi l'œuvre divine, elle n'induisait pas forcément de se livrer à une compétition avec Dieu. C'est ici ce qui est reproché à l'empereur qui, en cherchant à domestiquer les végétaux et l'eau pour créer un nouveau jardin d'Éden, en viendrait non plus à imiter respectueusement Dieu mais à tenter de l'égaler et, ainsi, faire montre d'une vanité sans borne. Il semble que le projet de Monomaque ait franchi une limite dans la perception de ses contemporains, surtout chez certains intellectuels qui avaient fréquenté son cercle proche, l'amenant à être perçu comme un homme faisant montre de démesure et même d'hybris, grave péché d'orgueil. Les sommes englouties par le projet, le grand nombre des poèmes célébrant la beauté de certains éléments, le faste des constructions et des cérémonies visant à glorifier ce grand

\footnotetext{
${ }^{39}$ Les empereurs étaient parfaitement conscients de ce qui était conforme ou non à la dignité impériale, c'est pourquoi la propagande impériale prenait toujours soin de souligner que chaque acte impérial était conforme à celle-ci. Voir Nike-Catherine Koutrakou, La propagande impériale byzantine. Persuasion et réaction (VIII $X^{e}$ siècles), Athènes, Université nationale d'Athènes, Faculté des lettres, 1994, p. 300301. Et même si la dimension guerrière de l'empereur restait importante à Byzance au cours de la première moitié du $\mathrm{XI}^{\mathrm{e}}$ siècle, le souverain devait surtout faire preuve de charité, apparaître en législateur et en protecteur des lettres et des arts. Face à la montée des périls pendant la seconde moitié du siècle et l'influence occidentale, la composante militaire de la fonction impériale prit une plus grande ampleur en particulier sous la dynastie des Comnènes (1081-1185).
} 
ouvrage, la forme des jardins aussi bien horizontale que verticale, tout tend à montrer que Constantin IX voulait créer un projet qui n'avait pas d'égal. Mais si son projet de jardins servait sa légitimité, il affaiblissait une des grandes vertus impériales depuis l'enracinement du christianisme dans l'Empire byzantin, l'humilité ${ }^{40}$.

Dans cette critique on retrouve des références christianisées aux descriptions de Suétone et de Tacite sur la Domus Aurea de Néron, avec ses longues galeries et ses piscines, ses champs et ses bois, ses espaces ouverts, au sein de la ville de Rome, qui blâmaient également le coût et les efforts nécessaires pour réaliser les volontés d'un souverain ${ }^{41}$. Face à une telle critique on peut penser que le portrait de Constantin IX avait pour objectif de faire une comparaison entre son comportement et les excès de $\mathrm{Néron}^{42}$, de manière à ternir l'image de l'empereur byzantin, et ce d'autant plus que la mort de ce dernier est, sous la plume du chroniqueur, directement liée à sa passion pour les jardins. En effet, Psellos, qui est le seul auteur à nous indiquer précisément la cause de la mort de Monomaque, rapporte qu'il aurait contracté une maladie pulmonaire au cours d'une baignade dans une des piscines qu'il s'était fait aménager au sein des Manganes. Constantin IX aurait donc été tué par son chef-d'œuvre, celui-là même qui l'avait conduit à tenter de rivaliser avec Dieu, une mort causée par la réprobation divine en raison de l'orgueil qu'il tirait de ses réalisations architecturales ${ }^{43}$.

\footnotetext{
40 Jouanno Corinne, "Réflexions sur pouvoir et démesure à Byzance », Kentron, $\mathrm{n}^{\circ} 23,2007$, p. 127-165.

${ }^{41}$ Sur ce sujet, voir Yves Perrin, « La Domus Aurea et l'idéologie néronienne », dans Le système palatial en Orient, en Grèce et à Rome. Actes du colloque de Strasbourg, 19-25 juin 1985, dir. Edmond Lévy, Leiden, Brill, 1987, p. 359-391.

${ }^{42}$ Henry Maguire, « Gardens and Parks in Constantinople », art. cit., p. 260.

${ }^{43}$ Psellos, Chronographie, éd. cit., t. II, 6, CCI. «Privé de notre réconfort, n'ayant plus pour le charmer la lyre de la raison, l'empereur chercha un refuge dans les amusements des sens. Ainsi, au milieu d'une prairie pleine de fruits de toute sorte, il
} 


\section{Un objet de mises en scène pour Monomaque}

Quand Psellos présente la politique d'empereur-jardinier de Constantin IX comme une œuvre néfaste et inutile, il nous livre sa vision du comportement de l'empereur et peut-être d'une partie de la cour. Toutefois, qu'en était-il vraiment? Quels étaient les objectifs de Monomaque? Pourquoi déployer autant de moyens pour constituer ce parc des Manganes? Il serait peu avisé de chercher à comprendre les motivations de l'empereur en les considérant simplement par une lecture sommaire de la source principale, la Chronographie de Psellos. En effet, en se penchant un peu plus sur ce texte, on se rend compte que l'opiniâtreté de Monomaque dans son rôle d'empereur-jardinier cherchait à démontrer sa dignité de lieutenant de Dieu sur terre par la création d'un paradis au sein de la capitale impériale. Il est d'ailleurs révélateur que Psellos établisse une comparaison entre les travaux de l'empereur pour assurer l'approvisionnement en eau de la capitale et l'un des fleuves sortis de l'Éden ${ }^{44}$.

fait creuser une piscine profonde ; il met de niveau avec le terrain le tour des bords et il y fait amener des courants d'eau. Si quelqu'un, ne sachant pas à l'avance que la prairie était creusée en son milieu, s'avançait sans méfiance pour cueillir une pomme ou une poire, il tombait dans l'eau, faisait un plongeon dans les profondeurs, et, revenant à la surface, se mettait à nager : l'événement était, pour l'empereur, un divertissement délicieux. Pour ne point s'en tenir à l'amusement avec la piscine, il bâtit un gracieux pavillon auquel il donne un entourage superbe ; mais alors, se mettant plusieurs fois par jour à nager dans l'eau chaude, sortant et rentrant, il attrape sans s'en apercevoir un coup d'air au côté. Tout d'abord, le trait ne le frappa pas précisément à fond ; mais ensuite, répandant le poison dans les viscères, il s'empara de la plèvre ». La principale occurrence qui mentionne l'installation d'une piscine dans un verger renvoie au Roman de Barlaam et de Josaphat, cette version chrétienne de la vie du Bodhisattva où la piscine sert au baptême du prince Josaphat. Une miniature d'un manuscrit $\mathrm{du} \mathrm{XIII}^{\mathrm{e}}-\mathrm{XIV}^{\mathrm{e}}$ siècle dépeint cette scène et présente un paysage sensiblement similaire à celui que décrit Michel Psellos. Voir Henry Maguire, «Imperial Gardens and the Rethoric of Renewal », art. cit., p. 184.

44 Michaelis Pselli. Orationes panegyricae, éd. George Dennis, Leipzig, B. G. Teubner, 1994, discours $\mathrm{n}^{\circ} 3,76-80$. «Il [Constantin $\mathrm{I}^{\mathrm{er}}$ ] a construit la cité qui porte son nom et l'a fortifié avec de puissants remparts, mais toi [Constantin IX] tu lui à davantage montré ta faveur car tu l'as embellie de magnifiques constructions; lui ayant même donné le fleuve qui sort de l'Eden, afin que la fleur de la cité ne puisse 
Pour les Byzantins, les jardins avaient de nombreuses utilités et dans le cas de jardins attachés aux palais impériaux, ceux-ci n'étaient pas uniquement destinés aux loisirs des empereurs mais avaient aussi pour fonction de servir de réceptacles à leurs idées politiques. Les jardins impériaux pouvaient servir de lieu de réception pour certaines ambassades. Ils avaient aussi, et surtout, avoir une fonction symbolique inscrite dans l'idéologie de leur constructeur. L'idée principale qui était véhiculée dans l'existence ou la création de jardins par l'empereur était l'association de ces jardins avec le renouveau impérial, porté par Monomaque: le jardin évoquait un nouveau printemps, un nouveau paradis, un nouveau monde. Le jardin impérial pouvait aussi servir de témoignage visuel et métaphorique des victoires renouvelées du souverain sur les bêtes sauvages qui figuraient les ennemis de l'Empire. En ce sens, l'aménagement horticole n'était pas en rupture avec les devoirs militaires de l'empereur ${ }^{45}$. C'était d'ailleurs une habitude rhétorique des panégyriques impériaux d'associer l'érection par un empereur d'un ensemble architectural ou végétal au principe de renouveau de l'Empire ${ }^{46}$. Cet oikos du jardin impérial indiquait le changement de règne, en particulier en l'accompagnant d'un très grand parc qui lui permettait de souligner que le pouvoir comme la nature s'épanouissait sous le gouvernement d'un nouvel empereur après l'intermède des souverains paphlagoniens (1034-1042).

La volonté de Monomaque était d'agir selon les procédés divins et de créer un paradis en remodelant le paysage qui était destiné à accueillir l'ouvrage impérial. Cet espace ne serait qu'abondance et devait être

pas se faner». Voir aussi Henry Maguire, «Imperial Gardens and the Rethoric of Renewal », art. cit., p. 181.

${ }^{45}$ Ibid., p. 182.

${ }^{46}$ Ibid., p. 187-189. L'idée apparaît dès le VII siècle au cours du règne d'Héraclius et se poursuit au cours du $\mathrm{X}^{\mathrm{e}}$ siècle sous la plume de Constantin VII, avant de revenir sur le devant de la scène avec Constantin Monomaque. 
exempt d'activités agricoles afin de protéger la sacralité de ce lieu qui avait vocation à enchâsser l'église, le monastère et le palais des Manganes. Cet écrin de verdure et d'ouvrages terrassés devaient servir à sublimer les bâtiments principaux.

L'aménagement des jardins par le pouvoir permettait de souligner que l'empereur était l'égal de Dieu sur terre par sa capacité à remodeler la nature, à lui donner la forme qu'il souhaitait tel un nouvel Éden ${ }^{47}$. Le fait, d'ailleurs, que Psellos fasse la comparaison entre Monomaque et le Créateur est assez éloquente, puisque dans sa critique de l'action de l'empereur, il traduit l'effet recherché par le souverain. Très souvent, les rhéteurs byzantins évoquaient les vertus, réelles ou attendues, de l'empereur par le biais de métaphores, les comparant à des fleurs ou à l'arrivée du printemps. Or la métaphore qui est le plus souvent utilisée dans ce registre lexical pour Constantin IX est l'astre solaire, dispensateur de vie. Michel Psellos est celui qui utilise le plus cette analogie, en comparant l'empereur au soleil printanier qui permettait à la nature de se ressourcer et de revenir à la vie, ce qui montre le double discours de Psellos en fonction de la période à laquelle il écrit ${ }^{48}$. En effet, alors qu'il est rhéteur au service de Constantin IX, il valorise l'œuvre de l'empereur dans le quartier des Manganes, capable tel un soleil de faire jaillir la vie, mais quand il écrit sa Chronographie après la mort de Monomaque, il ne fait que critiquer le gaspillage et la vanité du souverain. La description de

\footnotetext{
${ }^{47}$ Psellos, Chronographie, éd. cit., t. II, 6, CLXXV.

48 «Roi-Soleil : qui donc me reprocherait de vous adjoindre ce nom qui sied si bien à votre personne ? Car n'est-ce pas tant de l'orbite de vos vertus que du tour vivace de votre esprit, de la grandeur de votre nature et de l'éclat de votre beauté que vous illuminez la terre entière ? Mais que soient à la mesure de ma perception les rayons que vous lancez aujourd'hui : arrêtez-vous au milieu du jour, et montrez-vous à votre périgée, sans vous rendre inaccessible par les scintillements de votre lumière aurorale afin de ne pas éblouir de votre splendeur extrême. C'est une force plus divine que vous conférerez, et vous inciterez à regarder en face avec plus de confiance votre lumière ineffable.», trad. Sébastien Lüthi dans "Michel Psellos, Panégyrique 1: traduction Princeps et commentaire », Byzantion, vol. 77, 2007, p. 501-565.
} 
l'empereur à Byzance dans les panégyriques utilisait deux autres idées qui le reliaient à la nature. Le souverain était présenté comme un créateur, dont les actions par l'aménagement de jardins étaient parallèles à celles de Dieu, créateur de toutes choses, et les panégyristes décrivaient aussi le jardin impérial comme un paradis terrestre ${ }^{49}$. Le choix de Monomaque de recourir à une certaine végétalisation de l'image de son pouvoir n'était donc pas une innovation mais un héritage impérial séculaire. Pourtant, on ne peut nier qu'il a poussé cette tradition plus loin que la plupart des empereurs byzantins, en plaçant un jardin au cœur d'une opération de réaménagement urbain ${ }^{50}$.

L'objectif avoué, ou en tout cas assumé, de Constantin IX était ainsi de parvenir à une domestication des végétaux, lui permettant de montrer à ses sujets qu'il était capable de dompter la nature ${ }^{51}$ ce qui pouvait démontrer deux choses : d'une part sa capacité à gérer les affaire de l'empire et à vaincre les ennemis extérieurs, d'autre part, son aptitude à apporter l'abondance à son peuple puisqu'il pouvait transformer une région de Constantinople plutôt ingrate en un espace fertile et luxuriant.

\footnotetext{
${ }^{49}$ Henry Maguire, « Imperial Gardens and the Rethoric of Renewal », art. cit., p. 190.

${ }^{50} \mathrm{Si}$ l'on constate une végétalisation du pouvoir impérial sous Monomaque, il faudrait pourtant éviter de conclure que l'empereur a recouru à un usage similaire de la végétalisation, ou tout du moins aussi intense, dans sa pratique du pouvoir que l'a fait Auguste pour démontrer sa souveraineté. Sur cette question, les décorations de l'Ara Pacis sont particulièrement révélatrices du choix du premier empereur romain de faire usage d'ornements végétaux pour symboliser son autorité, Gilles Sauron, L'histoire végétalisée : ornement et politique à Rome, Paris, Picard, 2000. En effet, l'empereur n'a pas choisi d'éléments végétaux attitrés pour le représenter et il semble qu'il ait choisi de limiter la végétalisation à l'iconographie de certains objets de cour et au développement du parc des Manganes.

${ }^{51}$ La construction des Manganes, les jardins inclus, s'est déroulée de 1042 à 1047, c'est-à-dire pendant la première moitié du règne de Constantin IX et qui fut la plus réussie, puisque l'empereur fut capable de conquérir l'Arménie, de repousser deux invasions et d'abattre deux usurpateurs. L'achèvement des jardins représentait, en quelque sorte, l'acmé du règne de l'empereur, qui fut contraint de faire face à des guerres en Orient, dans les Balkans et en Italie à partir de 1048 et jusqu'à sa mort en 1055. Les jardins des Manganes lui servirent à magnifier sa politique jusqu'en 1047 et lui permirent sans doute d'entretenir l'idée qu'il était encore capable, par la suite, de maîtriser le monde, et donc son empire, en dominant le nouvel Éden.
} 
L'intérêt qu'exerçaient les jardins pour les membres de l'aristocratie ne pouvait que sortir renforcé d'une telle politique d'aménagement dans un contexte où, justement, des aristocrates montaient sur le trône et avaient grand besoin de se légitimer. L'ascension des héritiers des familles les plus prestigieuses au pouvoir pendant l'ensemble du XI siècle contribua sans doute à mêler les intérêts horticoles des empereurs et des aristocrates contribuant à un renouvellement de l'attrait pour les ouvrages naturels par les empereurs et dont Constantin Monomaque fut sans doute le plus éminent représentant.

Cherchant à soutenir sa légitimité et à faire la démonstration de son pouvoir au sein de Constantinople, Constantin Monomaque s'est employé à construire un grand parc impérial pour donner corps à son idéologie et diffuser l'image d'un souverain bienveillant mais aussi capable de dominer la nature et de la façonner selon ses besoins, assurant ainsi de façon métaphorique le renouveau de l'Empire. En menant à bien ce projet, le basileus a suivi les traces de ses prédécesseurs mais a aussi été capable d'introduire des nouveautés dans la conception de son jardin afin de souligner son unicité et, ainsi, magnifier son autorité. L'ampleur et le faste des travaux décidés par Constantin IX ont fait couler beaucoup d'encre dès son époque et jusqu'à une date récente. Les jardins des Manganes ont servi aux chroniqueurs médiévaux et aux historiens modernes à mettre en exergue le gaspillage des ressources financières de l'Empire par Monomaque. Une telle perception nous indique que l'entreprise de Constantin IX visant à asseoir sa légitimité était vue comme trop ambitieuse et, surtout, trop coûteuse par certains contemporains, au premier rang desquels Michel Psellos. Pourtant, l'inauguration de l'église de Saint-Georges-le-Tropaiophore en avril 1047, qui servit à célébrer la victoire de l'empereur sur le rebelle 
Tornikios dans le cadre des jardins des Manganes peut être considérée comme l'apogée de son règne, à la fois pour les affaires intérieures et extérieures. Elle montre l'importance politique que revêtaient ses jardins pour Constantin IX. L'empereur se fit d'ailleurs ensevelir dans le monastère de Saint-Georges-des-Manganes, situé à proximité de l'église du Tropaiophore et au sein du parc impérial, faisant ainsi de sa création végétale le lieu de son repos éternel. 
